\title{
Short course chemotherapy for tuberculous lymphadenitis in children
}

M S Jawahar, S Sivasubramanian, V K Vijayan, C V Ramakrishnan, C N Paramasivan, Vanaja Selvakumar, Sare Paul, S P Tripathy, R Prabhakar

\begin{abstract}
Objective-To assess the efficacy of a short course chemotherapy regimen for treating tuberculosis of the lymph nodes in children.

Design-Open, collaborative, outpatient clinical trial.

Setting-Outpatient department of the Tuberculosis Research Centre, paediatric surgery departments of the Institute of Child Health and Hospital for Children and the Government Stanley Hospital, Madras, South India.

Patients - Children aged 1-12 years with extensive, multiple site, superficial tuberculous lymphadenitis confirmed by biopsy (histopathology or culture).

Interventions-Patients were treated with a fully supervised intermittent chemotherapy regimen consisting of streptomycin, rifampicin, isoniazid, and pyrazinamide three times a week for two months followed by streptomycin and isoniazid twice a week for four months on an outpatient basis. Surgery was limited to biopsy of nodes for diagnosis and assessment.
\end{abstract}

Main outcome measures-Response to chemotherapy was assessed by regression of lymph nodes and healing of sinuses and abscesses during treatment and follow up. Compliance with treatment and frequency of adverse reactions were also estimated.

Results - 197 Patients were admitted to the study and 168 into the analysis. The regimen was well tolerated and compliance was good with $101(60 \%)$ patients receiving the prescribed chemotherapy within 15 days of the stipulated period of six months. Those whose chemotherapy extended beyond that period received the same total number of doses. Clinical response was favourable in most patients at the end of treatment. Sinuses and abscesses healed rapidly. Residual lymphadenopathy (exceeding 10 $\mathrm{mm}$ diameter) was present in $50(30 \%)$ patients at the end of treatment; these nodes were biopsied. Fresh nodes, increase in size of nodes, and sinuses and abscesses occurred both during treatment and follow up. After 36 months of follow up after treatment only $5(3 \%)$ patients required retreatment for tuberculosis.

Conclusion-Tuberculous lymphadenitis in children can be successfully treated with a short course chemotherapy regimen of six months.

Indian Council of Medical Research, New Delhi,

India

S P Tripathy, MD, additional director general

Correspondence to:

Dr Jawahar.

Requests for reprints to: $\mathrm{Dr}$ Prabhakar.

Br Med F 1990;301:359-62 pulmore is little inform about its value in lymph node disease in children. In controlled clinical trial in Britain the British Thoracic Society found that a nine month regimen of rifampicin and isoniazid supplemented by ethambutol for the first two months was as effective as a conventional 18 month regimen in adults with tuberculous lymphadenitis, ${ }^{56}$ and McCarthy and Rudd have reported the success of a six month regimen. ${ }^{7}$ Previous reports on the management of this disorder had dealt with conventional regimens of longer duration with or without surgery. ${ }^{8-10}$ All these studies were of adults. We report our experience with an intermittent six month regimen for treating children with predominantly multiple site, extensive, and severe lymphadenitis in Madras, South India, including the findings during 36 months of follow up after stopping treatment.

\section{Patients and methods}

The study was conducted at the Tuberculosis Research Centre, Madras (Indian Council of Medical Research) in collaboration with the paediatric surgery departments of the Institute of Child Health and Hospital for Children and the Government Stanley Hospital. Children aged 1-12 living in or near Madras with tuberculosis of the lymph nodes proved by biopsy (by histopathological examination or culture) were eligible for admission to the study. Those with renal, hepatic, or haematological disease or who had previously received more than one month of treatment for tuberculosis were not eligible.

\section{PRETREATMENT ASSESSMENT AND INVESTIGATIONS}

Children with a clinical diagnosis of tuberculous lymphadenitis were assessed initially by a paediatric surgeon, who performed a biopsy of the most prominent lymph node under general anaesthesia. Serial sections of the node were examined by an independent pathologist. Bacteriological investigations consisted of examination of a smear by fluorescence microscopy and culture of the node for tubercle bacilli using multiple media (Löwenstein-Jensen medium, Löwenstein-Jensen medium with sodium pyruvate, 7-hydroxy-11 oleic acid albumin medium, and liquid Kirchner's medium). "Growth of Mycobacterium tuberculosis in any one medium was considered diagnostic for tuberculosis. Positive cultures were identified and drug sensitivity tests were carried out using standard procedures. $^{12}$

Patients were admitted to the study if histological examination of the lymph node suggested tuberculosis. Patients whose node yielded $M$ tuberculosis on culture, but in whom histological examination of the node was negative were also admitted. All patients had a posteroanterior radiograph of the chest; a smear and culture for $M$ tuberculosis from sputum if the radiograph was abnormal, and from any sinus discharge; a Mantoux test with 1 tuberculin unit purified protein derivative RT23 with Tween 80; and examination of urine for albumin, sugar, urobilinogen, and acetylisoniazid. 
SHORT COURSE CHEMOTHERAPY REGIMEN

Patients were treated with a fully supervised intermittent regimen of six months' duration, consisting of streptomycin $(40 \mathrm{mg} / \mathrm{kg}$ body weight; maximum dose $750 \mathrm{mg}$ ), rifampicin (as syrup, $10 \mathrm{mg} / \mathrm{kg}$ ), isoniazid ( 15 $\mathrm{mg} / \mathrm{kg}$ ), and pyrazinamide $(45 \mathrm{mg} / \mathrm{kg}$ ) three times a week for two months followed by streptomycin and isoniazid twice a week for the next four months. A dosage schedule with $5 \mathrm{~kg}$ weight interval was used. Patients received the drugs as a single dose under supervision on an outpatient basis.

Missed doses were compensated so that a patient received 26 doses of the first phase (four drugs) within three months and 36 doses of the continuation phase (two drugs) within six months. Thus the maximum duration of treatment was nine months.

\section{ASSESSMENT DURING TREATMENT AND FOLLOW UP}

Clinical progress was monitored every month by a physician at the Tuberculosis Research Centre and by the paediatric surgeon at one, three, and six months and, if chemotherapy was prolonged beyond six months, at the end of treatment also. Radiography of the chest and sputum examinations were repeated at one, two, and six months if initially they had shown abnormal results. A specimen of urine was examined monthly for acetylisoniazid.

At the completion of treatment the surgeon had the option to repeat the lymph node biopsy if residual lymphadenopathy of more than $10 \mathrm{~mm}$ diameter was present. Histological and bacteriological examinations were done on these biopsy specimens. In the follow up period the patients were examined monthly up to 12 months, three monthly up to 24 months, and every six months thereafter.

TABLE I-Pretreatment characteristics of 168 children with tuberculous lymphadenitis

\begin{tabular}{|c|c|}
\hline & No (\%) of patients \\
\hline \multicolumn{2}{|l|}{ Sex: } \\
\hline Male & $85(51)$ \\
\hline Female & $83(49)$ \\
\hline \multicolumn{2}{|l|}{ Age (years): } \\
\hline $1-4$ & $78(46)$ \\
\hline $5-8$ & $41(24)$ \\
\hline $9-12$ & $49(29)$ \\
\hline \multicolumn{2}{|l|}{$\begin{array}{l}\text { Mantoux test ( } 1 \text { tuberculin unit purified protein } \\
\text { derivative) reaction: }\end{array}$} \\
\hline $0-9 \mathrm{~mm}$ & $14(8)$ \\
\hline $10-14 \mathrm{~mm}$ & $7(4)$ \\
\hline $15-19 \mathrm{~mm}$ & $40(24)$ \\
\hline$\geqslant 20 \mathrm{~mm}$ & $107(64)$ \\
\hline \multicolumn{2}{|l|}{ Chest $x$ ray finding: } \\
\hline Hilar adenitis & $17(10)$ \\
\hline Parenchymal shadows (tuberculous) & $6(4)$ \\
\hline Calcified spots & $16(10)$ \\
\hline Parenchymal shadows (non-tuberculous) & $10(6)$ \\
\hline Normal & $119(71)$ \\
\hline \multicolumn{2}{|l|}{ Bacteriological examination of lymph node } \\
\hline Culture positive & 96 \\
\hline Smear positive & $31\}(62)$ \\
\hline $\begin{array}{l}\text { Smear negative } \\
\text { Culture negative }\end{array}$ & $65 \int^{(02)} 60$ \\
\hline $\begin{array}{l}\text { Culture negative } \\
\text { Smear positive }\end{array}$ & 10! 60 \\
\hline Smear negative & $50\}(38)$ \\
\hline Not tested & 12 \\
\hline \multicolumn{2}{|l|}{ Histopathological examination of lymph node } \\
\hline Positive & $164(98)$ \\
\hline Negative & $4(2)$ \\
\hline
\end{tabular}

TABLE II-Incidence of new nodes, enlargement of existing nodes, and new sinuses and abscesses during treatment by short course chemotherapy of 168 children with tuberculous lymphadenitis

\begin{tabular}{lccccccccc}
\hline & & \multicolumn{5}{c}{ Month of occurrence } \\
\cline { 5 - 9 } & $\begin{array}{c}\text { No of } \\
\text { patients }\end{array}$ & $\begin{array}{c}\text { No of } \\
\text { episodes }\end{array}$ & 1 & 2 & 3 & 4 & 5 & 6 \\
\hline New nodes or enlargement of nodes & $32^{\star}$ & 36 & 14 & $\dagger$ & 11 & $\dagger$ & $\dagger$ & 11 \\
Sinuses & 10 & 10 & $6 \neq$ & 1 & 1 & 2 & & & 1
\end{tabular}

ॠ28 Patients had one episode each and four patients had two episodes each; assessment for lymph node enlargement was done at 1,3 , and 6 months.

tNot assessed.

$\ddagger$ Four of these patients had abscesses on admission.

\section{Results}

Intake to the study was from February 1980 to September 1985. Of the 197 patients admitted to the study, 29 were excluded from analysis (because of ineligibility for admission to study (16), isolation of atypical mycobacteria ( $M$ terrae complex-probably contaminants) on lymph node culture (6), defaulting before completing treatment (3), drug toxicity (2), and loss to follow up after treatment (2)). This report presents the findings in 168 patients who were followed up for 36 months after completing treatment.

Table I shows the pretreatment characteristics of the study population. In all, 96 patients had positive lymph node cultures, including four in whom the results on histopathological examination of the node were negative. Of the positive cultures, 18 (19\%) were resistant to one or more drugs: two to streptomycin, 10 to isoniazid, and six to both; none was resistant to rifampicin.

Multiple, extensive lymph node involvement was common. The mean number of node groups per patient was $2 \cdot 8$. In only $40(24 \%)$ patients was a single group of nodes affected, in $48(29 \%)$ two groups, in 11 $(7 \%)$ three, in $53(32 \%)$ four, and in $16(10 \%)$ five or more groups were affected. In $59(35 \%)$ patients only cervical nodes were affected, in $10(6 \%)$ only axillary nodes, and in $13(8 \%)$ only inguinal nodes. Sixty five (39\%) patients had a combination of cervical and axillary lymphadenitis, and in $18(11 \%)$ all three groups of nodes were affected. Three patients had adenitis at other sites (epitrochlear (two) and femoral (one)). Fourteen patients had sinuses, 26 had abscesses, and 23 had both.

\section{TREATMENT}

In all, $101(60 \%)$ patients had completed the chemotherapy within six months and 15 days. A further 32 (19\%) had chemotherapy extended up to seven months, $26(15 \%)$ up to eight months, and nine (5\%) up to nine months to compensate for missed doses.

All 168 patients improved clinically with treatment. Lymph nodes became smaller, though they did not always become impalpable. Sinuses and abscesses healed rapidly: of the 63 lesions, $51(81 \%)$ resolved by one month of treatment, $60(95 \%)$ by three months, and all by the end of treatment. During treatment, nodes enlarged or fresh nodes appeared in 32 patients (36 episodes), and fresh sinuses and abscesses occurred in 10 and four patients respectively (table II). One patient developed abscesses on three occasions.

At the end of treatment $50(30 \%)$ patients had residual lymphadenopathy $(>10 \mathrm{~mm})$, for which repeat biopsy was carried out. Of these, 41 biopsies were histologically and bacteriologically negative. Two patients had positive culture for $M$ tuberculosis (one also had positive results on histological examination); both cultures were fully sensitive to streptomycin, isoniazid, and rifampicin. Both patients were retreated for tuberculosis. $M$ scrofulaceum was isolated in one patient who was not treated again as she had only a single residual node which was removed at biopsy and she had no symptoms. Six patients with positive results only on histological examination were not treated. One of them developed a cold abscess two months later and was then retreated.

\section{FOLLOW UP}

All the patients were followed up for 42 months from the start of treatment (33-36 months after treatment). Enlargement of nodes and appearance of new nodes, sinuses, and abscesses occurred in this period (table III). Six patients were subjected to further biopsies to exclude relapse: one at five months, another at seven months, two at nine months, and two more at 24 months after stopping treatment. All the biopsies were 
TABLE III - Events during follow up of 168 children treated with short course chemotherapy for tuberculous lymphadenitis

\begin{tabular}{|c|c|c|c|c|c|c|c|c|c|c|c|c|}
\hline & \multirow{2}{*}{$\begin{array}{c}\text { No of } \\
\text { patients }\end{array}$} & \multicolumn{11}{|c|}{ Month of occurrence } \\
\hline & & 7 & 8 & 9 & 12 & 15 & 18 & 21 & 24 & 30 & 36 & 42 \\
\hline New nodes or enlargement of nodes & $26^{\star}$ & $\dagger$ & $\dagger$ & 10 & 4 & 8 & 5 & 6 & 2 & 3 & & 1 \\
\hline Sinuses & 5 & 2 & & 2 & & 1 & & & & & & \\
\hline Abscesses & $7^{\bullet}$ & & $1 \neq$ & & 1 & 2 & 1 & 1 & & 1 & & \\
\hline Mesenteric adenitis & 1 & & & 1 & & & & & & & & \\
\hline Tuberculous meningitis & 1 & & & & & & & & & & & 1 \\
\hline
\end{tabular}

^19 Patients had one episode, three patients had two, three had three, and one had five episodes; total 39 episodes. †Not assessed.

†Cervical cold abscess; retreated for tuberculosis.

negative histologically and bacteriologically. Sinuses that appeared also healed with a course of ampicillin or co-trimoxazole. One patient developed mesenteric lymphadenitis four months after stopping treatment and another tuberculous meningitis 35 months after stopping treatment.

In all, five $(3 \%)$ patients required retreatment for tuberculosis after completing the study regimen, two because of a positive node culture at the end of treatment, one for a cervical cold abscess, another for mesenteric lymphadenitis, and the last for tuberculous meningitis. In all five patients pretreatment culture had yielded organisms sensitive to streptomycin, isoniazid, and rifampicin.

\section{ADVERSE REACTIONS TO DRUGS}

In all, 178 patients completed treatment, including 10 patients not included in the main analysis: six who were not eligible for admission to the study, two lost to follow up, and two in whom streptomycin was toxic, for which the regimen was changed. Reactions possibly attributable to drugs were observed in 13 other patients; most were mild. Four patients had giddiness due to streptomycin so the dosage was reduced. Two patients developed jaundice; treatment was withheld temporarily and resumed uneventfully once the jaundice had subsided. Four patients had gastrointestinal symptoms and three pruritus; these symptoms responded to treatment.

\section{Discussion}

A striking feature of this study was the severity with which lymph nodes were affected. Multiple sites were very common, and in $69(41 \%)$ patients four or more groups of nodes were affected. Cervical and axillary adenitis occurred together in $65(39 \%)$ and cervical adenitis alone in 59(35\%) patients, and all three groups of nodes (cervical, axillary, and inguinal) were affected in $18(11 \%)$ patients. Advanced lesions with sinuses or abscesses, or both, were seen in $63(38 \%)$ of the 168 patients, a much higher figure than reported previously. ${ }^{913}$

The overall bacteriological positivity rate (smear and culture) from the lymph node biopsy specimens was $68 \%$. This compares favourably with the figures reported in other studies, which have ranged from 30 $55 \% .{ }^{91314} \mathrm{We}$ used multiple culture media as recommended by Mitchison et al. ${ }^{11}$

Clinical response to treatment was favourable in most patients, with regression in the size of lymph nodes and prompt healing of sinuses and abscesses. The rapidity with which sinuses and abscesses resolved was particularly striking, $51(81 \%)$ of these lesions having disappeared within one month of the start of treatment. Although lymph nodes became smaller, they did not always become impalpable. At the end of treatment, $50(30 \%)$ patients had one or more residual nodes exceeding $10 \mathrm{~mm}$ diameter. This is considerably higher than the $9 \%$ and $13 \%$ reported in the two earlier British studies $^{59}$ and is not surprising considering the extensive and severe type of disease seen in our population.

Both the chemotherapy and the follow up periods were punctuated by events such as enlargement of nodes and appearance of new nodes and of sinuses and abscesses in some patients. These events occurred relatively more frequently in the early months of chemotherapy and follow up and less often later. These episodes almost always resolved spontaneously or after a course of ampicillin or co-trimoxazole and did not presage a relapse. Similar observations have been documented by the British Thoracic Society study and by Campbell and Dyson. ${ }^{59}$ These transient events may be the result of cellular reaction to the products of disintegration of the bacilli, probably to their cell wall components. ${ }^{10}$ is Further, histological changes in the nodes may take a number of months to regress, as evidenced by the fact that six patients still had histological evidence of tuberculosis in the lymph node with negative cultures at the end of treatment.

In all, five (3\%) patients received additional chemotherapy for tuberculosis after completing the prescribed regimen. In two this was because they had positive lymph node cultures at the end of treatment, evidence that the regimen had not sterilised the nodes. Two patients had episodes of lymphadenitis (one cervical and one mesenteric) and another developed meningitis 35 months after stopping treatment.

The regimen was well tolerated. Apart from two patients who required a change of regimen for streptomycin toxicity (these patients were excluded from this analysis), only minor adverse reactions were experienced and treatment could be continued uninterrupted in most of the patients.

In conclusion, this study shows that a short course regimen of six months is effective in treating severe forms of lymph node tuberculosis in children. At the end of 36 months of follow up after treatment, the outcome was satisfactory in $163(97 \%)$ of the 168 patients. Increase in the size of nodes and appearance of new nodes, sinuses, and abscesses were seen during both treatment and follow up. These were usually transient events and did not suggest a relapse. Surgery was seldom indicated except for diagnosis and for draining lymph node abscesses in a few patients.

The patients are being followed up further and will be reported on after five years of follow up.

We thank paediatric surgeons Drs M S Ramakrishnan, T Durairajan, C K Annamalai, S Sridharan, M Ganapathy, Rajkishore Bagdi, G Kumaravel, T M Ramanujam, Ramanathan; medical officers in the Tuberculosis Research Centre Drs R Parthasarathy, O Nazareth, T Santha Devi, Rani Balasubramanian, S V Rathnasabapathy, D C Arumainayagam, R V S N Sarma, Rajeswari Ramachandran S Ramakrishnan, H B Chandrasekar, Reetha Vijayan, Rema Mathew, Pauline Joseph, R Balambal, and K C Umapathy Mr P Venkatesan for help with statistical analysis; staff of the social work department of the Tuberculosis Research Centre Mrs Sudha Ganapathy, Miss T V Mathibooshanam, Mrs Mangalagowri Krishnan, Mrs Sheila Fredricks, Mrs Geetha Shanmugam, Mrs Nirupa Charles, Mrs K Thilakavathy, Mrs Beena Thomas, and Mrs Meenalochani Dilip, and nursing staff under the guidance of Mrs J Monga and Mrs P Raghavan; Professors Wallace Fox and D A Mitchison, and Mr P R Somasundaram for help in the preparation of this manuscript and Mr R S Sen, Mrs Santha Sriraghavan, and Mr $P$ Karthigayan for secretarial assistance.

1 Medical Research Council Tuberculosis and Chest Diseases Unit. Nationa Survey of tuberculosis notifications in England and Wales in 1983: characteristics of disease. Tubercle 1987;68:19-32.

Murthy TVM. Tuberculous lymphadenitis in children. Indian Paediatr 1976;13:7.

3 Fox W. Whither short course chemotherapy? Br 7 Dis Chest 1981;75:331-57.

4 Tripathy SP. Short course chemotherapy of pulmonary tuberculosis. Indion fournal of Tuberculosis 1982;29:3-13

5 British Thoracic Society Research Committee. Short course chemotherapy for tuberculosis of lymph nodes: a controlled trial. Br Med f 1985;290:1106-8.

.


6 British Thoracic Society Research Committee. Short course chemotherapy for lymph node tuberculosis: final report at 5 vears. $\mathrm{Br} 7$ Dis Chest 1988:82: $282-4$

McCarthy OR, Rudd RM. Six month chemotherapy for lymph node tuberculosis. Respiratory Medicine 1989;83:425-7.

8 Gillam PMS, Knowles JP. The treatment of tuberculous lymphadenitis Tubercle 1963;44:112-8.

9 Campbell IA, Dyson AJ. Lymph node tuberculosis: a comparison of various methods of treatment. Tubercle 1977;58:171-9.

10 Campbell IA, Dyson AJ. Lymph node tuberculosis: a comparison of treatments 18 months after completion of chemotherapy. Tubercle 1979;60 95-8.

11 Mitchison DA, Allen BW, Devi Manickavasagar. Selective Kirchner medium in the culture of specimens other than sputum for mycobacteria. F Clin Pathol 1983:36:1357-61.

12 Canetti G, Fox W, Khomenko R, et al. Advances in the techniques of testing mycobacterial drug sensitivity and the use of sensitivity tests in tuberculosis control programmes. Bull WHO 1969;41:21-43.

13 Pamra SP, Mathur GP. A co-operative study of tuberculous cervical lymphadenitis. Indian f Med Res 1974;62:1631-46.

14 Kulkarni KG. Bacteriological study of tuberculous lymphadenitis. Indian foumal of Tuberculosis 1974;21:60-84.

15 Anonymous. Paradoxical responses during the chemotherapy of tuberculosis [Editorial]. F Infect 1987;15:1-3.

(Accepted 13 fune 1990)

\title{
Symptoms of low blood pressure: a population study
}

\author{
Simon Wessely, Judith Nickson, Brian Cox
}

\begin{abstract}
Objective-To establish whether an association exists between blood pressures in the "low normal" range and common symptoms such as tiredness, dizziness, headache, and palpitation, as suggested by French and German medical practice but not English or American medical practice.
\end{abstract}

Design-Cross sectional population based survey (the health and lifestyle survey) of blood pressure measurements and self reported common symptoms. Results were analysed by combined stratification and logistic regression.

Subjects-7383 (82\%) Adults aged 18 and over chosen from the electoral register in England, Wales, and Scotland for the health and lifestyle survey, in whom satisfactory physiological measurements were taken, from 9003 in the interviewed sample.

Main outcome measures-Body mass index, smoking, social class, exercise, self declared physical illnesses, hours slept, use of drugs, and psychological illness as determined with the general health questionnaire assessed as potential confounders.

Results-True confounders were sex, age, taking of drugs, physical illness, exercise, and body mass index. A negative association was found between systolic blood pressure and self reported tiredness and feeling faint, which persisted after adjustment for the confounders. The association was strongest in women aged under 50 . A negative association between systolic blood pressure and headache and a positive association between systolic blood pressure and palpitation were explained by confounding by age.

Conclusions - Systemic hypotension is associated with persistent tiredness but treatment is not suggested as either possible or necessary. Instead, low blood pressures may be associated with opposite effects on mortality contrasted with morbidity.

\section{London SE5 8AF}

Simon Wessely, MRCPSYCH, research fellow

\section{Office of the Regius Professor of Physic, University of Cambridge Clinical School, Cambridge Judith Nickson, BA, research associate}

\section{Department of}

Community Medicine,

University of Cambridge, Cambridge

Brian Cox, PHD, lecturer in

community medicine

Correspondence to:

Dr Wessely. even in the English speaking world, large numbers of people receive treatment for low blood pressure; one Canadian survey found that nearly a tenth of a community sample was receiving treatment for "low blood pressure."

What is the evidence? At the beginning of this century most authorities assumed that low blood pressure was associated with several so called "neurasthenic" symptoms, such as tiredness, weakness, fainting, and dizziness, ${ }^{67}$ although the same symptoms were also attributed to high blood pressure. ${ }^{8}$ However, scepticism increased, culminating in Robinson's definitive article on the topic in $1940 .{ }^{9} \mathrm{He}$ vehemently attacked those who regarded low blood pressure as pathological and presented data showing that low blood pressure was associated with decreased mortality. He continued, "the symptoms usually ascribed to hypotension are in reality commoner among hypertensive persons. There are no symptoms peculiar to low blood pressure." This rapidly became the new conventional wisdom. A leading text now states: "Most persons with systolic pressures in the range of 90 to $110 \mathrm{~mm} \mathrm{Hg}$ are normal and may actually have a greater life expectancy than those with 'normal' pressures."10 Although the evidence is, however, unequivocal that low blood pressure is associated with decreased mortality ${ }^{11}$ (the exception being the association between very low pressures and increased mortality in subjects with ischaemic heart disease, the so called $\mathrm{J}$ curve relation ${ }^{12}$ ), few data on the morbidity of low blood pressure exist. For example, although discussing mortality and morbidity, Robinson presented data only on mortality. ${ }^{9}$

Pemberton's analyses on data from an Australian survey, which disclosed a relation between tiredness and low blood pressure in women, ' reopened the debate. The relation did not extend to either dizziness or fainting, symptoms also traditionally associated with low blood pressure, whereas palpitations showed an opposite trend, being more common with higher blood pressures. Pemberton could quote only crude rates. Blood pressure and tiredness are influenced by many variables so it is necessary to determine whether his findings may not only be replicated but also be explained by confounding. We present an analysis of data from the health and lifestyle survey, ${ }^{13}$ which provides an opportunity to answer these questions.

\section{Methods}

\section{HEALTH AND LIFESTYLE SURVEY}

The health and lifestyle survey is the most comprehensive population based health survey carried out in Britain to date. The sampling frame for the survey was the entire adult population of England, Wales, and Scotland aged 18 and over. Preliminary selection was 